Perhaps the best balanced survey of all is that on trauma to the central nervous system by the well known Courville: this is a splendid piece of carefully balanced writing and tends to reflect the often inadequate or unbalanced treatment of other sections-for example, industrial disease, which picks out only asbestosis and caisson disease; or neonate and infant death, which gives the "battered baby" wholly disproportionate space-and no mention of syncytial virus infections.

Much good - and quite a lot of poor-reading lies within these seven hundred pages. The whole subject is treated with enthusiasm-something interesting is there for everyone and there is an excellent index. At $£ 8$ 15s. the book is plainly intended for the specialist and the library, and it deserves a place with each. I think Gradwohl would be content to see his book transmogrified in this way.

KEITH Simpson

\section{RUSSIAN GENERAL PHYSICS}

\section{General Physics}

Mechanies and Molecular Physies. By L. D. Landau, A. I. Akhiezer and E. M. Lifshitz. Translated by J. B. Sykes, A. D. Petford and C. L. Petford. Pp. $x+372$. (Oxford, London and New York: Pergamon Press, 1967.) 50s.; \$8.

THere are already quite a large number of excellent textbooks on general physics. However, there are so many diverse ways of teaching physics that there is still scope for new books with different approaches to the subject, although this is such a competitive field, with high rewards for the most successful, that new books need to be at least as good as the existing ones in their general style and content. In particular, a book on general physics needs to be fairly large. There will rarely be a close correspondence between the material in a particular book and the material which is taught in a particular course: a successful book needs to contain an excess of material so that different selections are possible.

The main weakness of this new addition to the field is in fact its size: the amount of material it contains is sufficiently small for it to be useful for only a particular type of general physies course. In its own way, it is a very good book: Landau and Lifshitz are already well known in the world of textbooks for their excellent Course of Theoretical Physics, and the standard of writing in this more elementary book is just as high. As might be expected from these authors, the approach is different from most textbooks on general physics, especially in the way that physical chemistry and crystallography are inter-related with the basic concepts of physics. In fact, the book will probably be most useful, not in courses on pure physics, but in chemical physies and other courses with a chemical flavour.

The book is intended for first and second year undergraduates, although most parts seem rather elementary for most British second year courses. Mechanics, heat and thermodynamics, and solid state physics are well covered, and in addition there is some elementary chemical physics and a rather curious chapter on the theory of symmetry, the contents of which are not necessary for an understanding of the rest of the book. The style is coherent and interesting, but is in no way urgent and modern as is the style of its American competitors; in fact the book was first written in 1937, and this original text, together with notes from some of Landau's lectures (published in 1948), have formed the basis of the present published text. Unfortunately, there are no problems or examples to augment and illustrate the material, again in strong distinction to modern textbooks on general physics. The translation into English is clear and unambiguous, although it does seem a little too formal and stodgy at times.
The book will almost certainly not have the wide appeal enjoyed by some of the texts designed for American physics courses, but it has a distinctive style and content which may well suit it to a few courses in physical science or chemical physics.

F. H. READ

\section{COSMIC RAYS AND SPACE PHYSICS}

\section{Cosmic Rays and Problems of Space Physics}

Edited by Yu. G. Shafer. (Proceedings of the AllUnion Conference. Yakutsk, August-September 1962.) Pp. vii +311. (Jerusalem: Israel Program for Scientific Translations; London: Macdonald-Oldbourne Press, 1967.) $90 s$.

THIs book contains the proceedings of the First All-Union Conference on Space Physics Aspects of Cosmic Ray Research held at Yakutsk in 1962. Because of the strength of cosmic ray research in the Soviet Union, the proceedings read like those of a full international conference and all aspects of the field are covered, ranging from geophysical effects to cosmological problems. There are thirty-seven research and introductory papers in full, and a few more are in synopsis form, an example of the latter being Ginzburg and Syrovatskii on the cosmic ray origin problem. The work of these authors, however, is already well known in English translation.

Among the useful introductory papers is the review by Pontecorvo on neutrinos and astrophysics. Properties of neutrinos are discussed and the importance of neutrino processes in astrophysics is emphasized. At some stages in stellar evolution the energy emitted into space as neutrino-antineutrino pairs becomes comparable to the energy emitted as photons. The type of neutrino emitted depends on stellar temperature and in principle information on nuclear processes deep inside the Sun can be obtained if the neutrino flux at the Earth can be studied.

Various theoretical papers to which it was otherwise difficult to refer are collected in the book. Among these is the independent work of both Krymskii and Shishov on the diffusion model for solar proton propagation in interplanetary space under a predominantly uni-directional magnetic field. Previous work considered only isotropic diffusion models and these Russian papers were probably the first to treat the more realistic anisotropic diffusion case. Dorman and co-workers develop formula for computing coupling coefficients in the high-energy range, relating the primary cosmic ray intensity at a given energy to the secondary intensity deep in the atmosphere. Estimation of these coefficients above the energy where they can be obtained empirically from the latitude effect remains a current problem. Pomanskii and Dovzhenko calculate the radiation length for bremsstrahlung and pair production for a large number of common substances. The effects of total screening, bremsstrahlung and pair production in the field of atomic electrons and of inaccuracies in the Born approximation are all taken into account.

Many of the quoted experimental results, such as the Sputnik 6 heavy nuclei data and the Cerenkov light shower data obtained in Crimea which established an upper limit to the high energy photon flux from possible sources, have already been well publicized. The proceedings, however, provide a useful reference for the results obtained at that time from the Moscow extensive air shower array and the Yakutsk underground telescope, both of which are experiments which continue to supply extensively used data. The last part of the book is devoted to instrumental techniques.

Apart from the fascinating overall picture provided of cosmic ray research in the Soviet Union, the book is mainly of use as a reference for research workers, although the student entering the field will obtain something of value from the review articles. 